\title{
JNK pathway mediates curcumin-induced apoptosis and autophagy in osteosarcoma MG63 cells
}

\author{
YAOWU ZHANG, PINGBO CHEN, HANGANG HONG, LEI WANG, YUBO ZHOU and YI LANG \\ Department of Orthopedic Surgery, Traditional Chinese Medicine Hospital of Xinjiang \\ Uyghur Autonomous Region, Urumqi, Xinjiang 830001, P.R. China
}

Received April 18, 2016; Accepted January 6, 2017

DOI: $10.3892 /$ etm.2017.4529

\begin{abstract}
Human osteosarcoma is a common primary malignancy of the bone in children and adolescents. It has been reported that curcumin is able to induce apoptosis in osteosarcoma MG63 cells through the mitochondrial pathway. However, whether curcumin is able to induce autophagy and the interaction between apoptosis and autophagy in osteosarcoma cells has yet to be fully elucidated. In the current study, it was determined that curcumin was able to significantly induce apoptosis, and lead to autophagy in MG63 cells. Notably, inhibition of apoptosis enhanced curcumin-induced autophagy due to upregulation of the c-Jun N-terminal kinase (JNK) signaling pathway. This finding was confirmed by the use of JNK-specific inhibitor, SP600125. Furthermore, our data showed that curcumin-induced apoptosis was increased when autophagy was completely inhibited by 3-methyladenine in MG63 cells. These results suggest that autophagy may have an important role in resistance to apoptosis when MG63 cells are incubated with curcumin. Thus, these results provide important insights into the interaction between apoptosis and autophagy in osteosarcoma cells and clinical treatment strategies using curcumin.
\end{abstract}

\section{Introduction}

Osteosarcoma is one of the most common primary malignant neoplasms in children, adolescents, and young adults (1). The introduction of chemotherapy has lead to a significant

Correspondence to: Dr Hangang Hong, Department of Orthopedic Surgery, Traditional Chinese Medicine Hospital of Xinjiang Uyghur Autonomous Region, 116 Huanghe Road, Xinjiang 830001, P.R. China E-mail: hangang_hong@126.com

Abbreviations: JNK, c-Jun N-terminal kinase; 3-MA, 3-methyladenine; MDC, monodansylcadaverine; ATG5, autophagy related 5; ERK, extracellular signal-regulated kinase; LC3, microtubule associated protein 1 light chain 3

Key words: osteosarcoma, apoptosis, curcumin, autophagy improvement in the prognosis of patients with localized osteosarcoma, and long-term survival rates of $<20 \%$ have been observed to improve to $>65 \%$ after the use of multiagent chemotherapy regimens (2). However, patients with osteosarcoma who present with metastasis continue to have poor prognosis, which is associated with a strong resistance to chemotherapy $(3,4)$. Consequently, it is imperative that novel treatment strategies are developed for such patients.

Curcumin (Fig. 1) is derived from the rhizome of the East Indian plant Curcuma longa. Over the past three decades, curcumin has been widely used as a cancer chemotherapy agent in a wide range of cancer models, including those for colorectal, pancreatic, breast and hematological malignancies, and has been utilized for its ability to alleviate therapy-induced toxicities such as Mitomycin C associated side-effects and chemotherapy-induced mucosal barrier injury (5-10). Curcumin has been found to inhibit the tumorigenesis and progression of various tumors, such as colorectal cancer, lung adenocarcinoma (5). These anti-cancer effects are predominantly mediated through the negative regulation of various oncogenic molecules and pathways, including activator protein 1 , nuclear factor $\kappa \mathrm{B}$, peroxisome proliferator-associated receptor gamma, signal transducer and activator of transcription, Wnt/ $\beta$-catenin, nuclear factor (erythroid-derived 2)-like 2, tumor necrosis factor- $\alpha$, interleukins, inducible nitric oxide synthase, cyclooxygenase-2, lipooxygenase, p38 mitogen-activated protein kinase (MAPK), c-Jun N-terminal kinase (JNK1/2), extracellular signal-regulated kinase $1 / 2$, growth factor induced signaling cascades, cyclin D1, p53, in addition to intracellular adhesion molecule-1 (11). Curcumin has also been used in combination with various other anti-cancer agents, such as gemcitabine, docetaxel and acetylcysteine in pre-clinical cancer studies $(5,10,12)$.

Autophagy, which is a process involving self-degradation and the turnover of cellular components, has a complex role in the initiation and progression of cancer. Evidence suggests that autophagy has anti-survival characteristics, and can contribute to tumor suppression in different cancer cell types (13). The predominant function of the JNK signal transduction pathway is to induce defense mechanisms that protect organisms against a number of stressors, including UV irradiation and oxidative stress, which can induce apoptosis or growth inhibition. This pathway has also been confirmed to be associated with the molecular events involved in the regulation of autophagy (14). 
The current study aimed to determine whether curcumin was able to induce autophagy in osteosarcoma cells. In addition, the underlying interaction between autophagy and apoptosis was investigated.

\section{Materials and methods}

Cell lines and curcumin. The human osteosarcoma cell line MG63 was purchased from the American Type Culture Collection (Manassas, VA, USA). All of the cells were cultured with Dulbecco's modified Eagle's medium (DMEM; Thermo Fisher Scientific, Inc., Waltham, MA, USA) supplemented with 10\% fetal bovine serum (FBSand penicillin/streptomycin (both Thermo Fisher Scientific, Inc.).

Proliferation assay. Cell proliferation assays were performed using cell counting kit (CCK)-8 (Dojindo Molecular Technologies, Inc., Rockville, MD, USA) in accordance with a previously described method (15). A total of 2,000 cells were seeded in each well in a 96-well plate. CCK-8 solution (10 $\mu$ l) was added to $100 \mu l$ of culture media, and the optical density was measured at $450 \mathrm{~nm}$. The concentrations of z-VAD-FMK, 3 -MA or SP600125 used in the experiments were 5,5 or $10 \mu \mathrm{M}$, respectively. Three independent experiments were performed.

Apoptosis assay. Cell apoptosis assays were performed by flow cytometry (BD FACSCalibur flow cytometer; BD Biosciences, Franklin Lakes, NJ, USA), as previously described $(16,17)$. Cells at a density of $0.5 \times 10^{5}$ cells/dish in $60-\mathrm{mm}$ cell culture dishes were pre-incubated with or without agents for $24 \mathrm{~h}$, collected and stained using the Annexin V-FITC Apoptosis Detection kit (BD Biosciences) according to the manufacturer's instructions. In brief, cells were washed with cold PBS and resuspended in $100 \mu \mathrm{l}$ Annexin V binding buffer, followed by incubation with fluorescein isothiocyanate-conjugated Annexin $\mathrm{V}$ and propidium iodide for $15 \mathrm{~min}$ at room temperature in the dark.

Western blot analysis. Total protein $(\sim 500 \mathrm{mg})$ was extracted using lysis reagents (Cell Signaling Technology, Inc., Danvers, MA, USA) and quantified using a bicinchoninic acid assay kit (Pierce; Thermo Fisher Scientific, Inc.). Proteins (50 mg) were separated by $10 \%$ SDS-PAGE and transferred to polyvinylidene difluoride (PVDF) membranes. The membranes were then blocked with 5\% skimmed milk for $2 \mathrm{~h}$ at room temperature and incubated overnight at $4^{\circ} \mathrm{C}$ with mouse anti-Bcl-2 monoclonal antibody (1:1,000; sc-56015), rabbit anti-caspase-3 polyclonal antibody $(1: 1,000 ;$ sc-271757), mouse anti- $\beta$-actin monoclonal antibody $(1 ; 1,000 ;$ sc- 81178 ; all Santa Cruz Biotechnology, Inc., Dallas, TX, USA), rabbit anti-Bax monoclonal antibody (1:1,000; ab-32503), rabbit anti-ATG5 monoclonal antibody (1:1,000; ab108327), rabbit anti-LC3-I/II polyclonal antibody (1:1,000; ab128025), rabbit anti-p-EKR polyclonal antibody $(1: 1,000$; ab176660), rabbit anti-p-JNK polyoclonal antibody (1:1,000; ab47337), rabbit anti-p-P38 polyclonal antibody $(1: 1,000 ;$ ab47363) and mouse anti-p-p53 polyclonal antibody (1:1,000; ab1431) (all Abcam, Cambridge, MA, USA) overnight at $4^{\circ} \mathrm{C}$ and further incubated for $1 \mathrm{~h}$ with horseradish peroxidase-conjugated anti-mouse $(1: 1,000 ; \mathrm{sc}-2371)$, or anti-goat $(1: 1,000 ; \mathrm{sc}-2350)$ or anti-rabbit

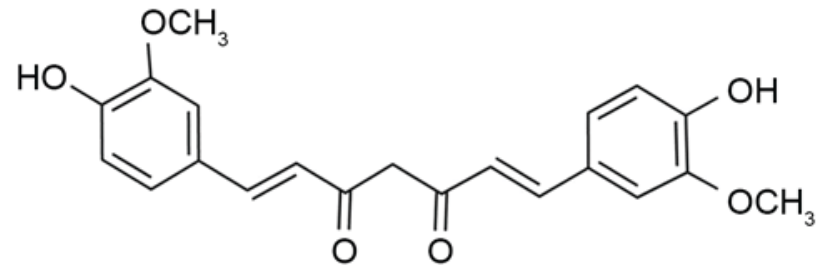

Figure 1. Structure of curcumin. (E, E)-1,7-bis(4-hydroxy-3-methoxyphenyl)1,6-heptadiene-3,5-dione diferuloylmethane diferulylmethane.

(1:1,000; sc-2370) secondary antibodies (all Santa Cruz Biotechnology, Inc.). Immune complexes were then detected by incubating the PVDF membranes with HRP-conjugated secondary antibody for $2 \mathrm{~h}$ at room temperature, followed by exposure of the membrane to enhanced chemiluminescence reagents (Pierce; Thermo Fisher Scientific, Inc.). Quantification of the protein bands was performed using Image J 1.48 software (National Institutes of Health, Bethesda, MD, USA).

Autophagy assay. Monodansylcadaverine (MDC; SigmaAldrich; Merck Millipore, Darmstadt, Germany), a specific marker for autophagic vacuoles was used in order to further confirm whether curcumin was able to induce autophagy. MG63 cells were labeled with $0.05 \mathrm{mM}$ MDC in PBS at $37^{\circ} \mathrm{C}$ for $10 \mathrm{~min}$, washed three times with PBS and immediately analyzed under an AV300-ASW confocal microscope (Olympus Corp., Tokyo, Japan) with a x60 oil lens. The amount of LC3 puncta per cell was quantified. A minimum of 10 cells in five independent experiments were analyzed at random.

Statistical analysis. Statistical analysis was performed using SPSS statistical software (version 16.0; SPSS, Inc., Chicago, IL, USA). Student's t-test was used to analyze all other data. All tests were two-tailed and $\mathrm{P}<0.05$ was considered to indicate a statistically significant difference.

\section{Results}

Curcumin suppresses osteosarcoma cell proliferation. To determine the cytotoxic effects of curcumin (Fig. 1) on osteosarcoma cell lines, a CCK- 8 assay was performed to evaluate the proliferation of human osteosarcoma MG63 cells. As shown in Fig. 2A, curcumin treatment markedly decreased the proliferation of MG63 cells in a dose- and time-dependent manner. Furthermore, the exposure of MG63 cells to curcumin at various concentrations for $24 \mathrm{~h}$ dose-dependently increased the number of cytolytic cells (Fig. 2B). The $\mathrm{IC}_{50}$ value for curcumin was $9.2 \mu \mathrm{M}$ in MG63 cells.

Curcumin promotes apoptosis in osteosarcoma cells. Flow cytometry was employed to investigate the anti-cancer effects of curcumin on the apoptosis of MG63 cells. Following treatment with different concentrations of curcumin $(1,5$ or $10 \mu \mathrm{M})$ for $24 \mathrm{~h}$, the apoptosis rate of cells was markedly increased in a dose-dependent manner (Fig. 3A). The Annexin V positive cell ratios (\%) for the concentrations of 1,5 or $10 \mu \mathrm{M}$ curcumin were 4.6, 23.8 and 41.9\%, respectively in MG63 cells. The results showed that 5 or $10 \mu \mathrm{M}$ curcumin could significantly induce MG63 cell apoptosis compared with the DMSO 

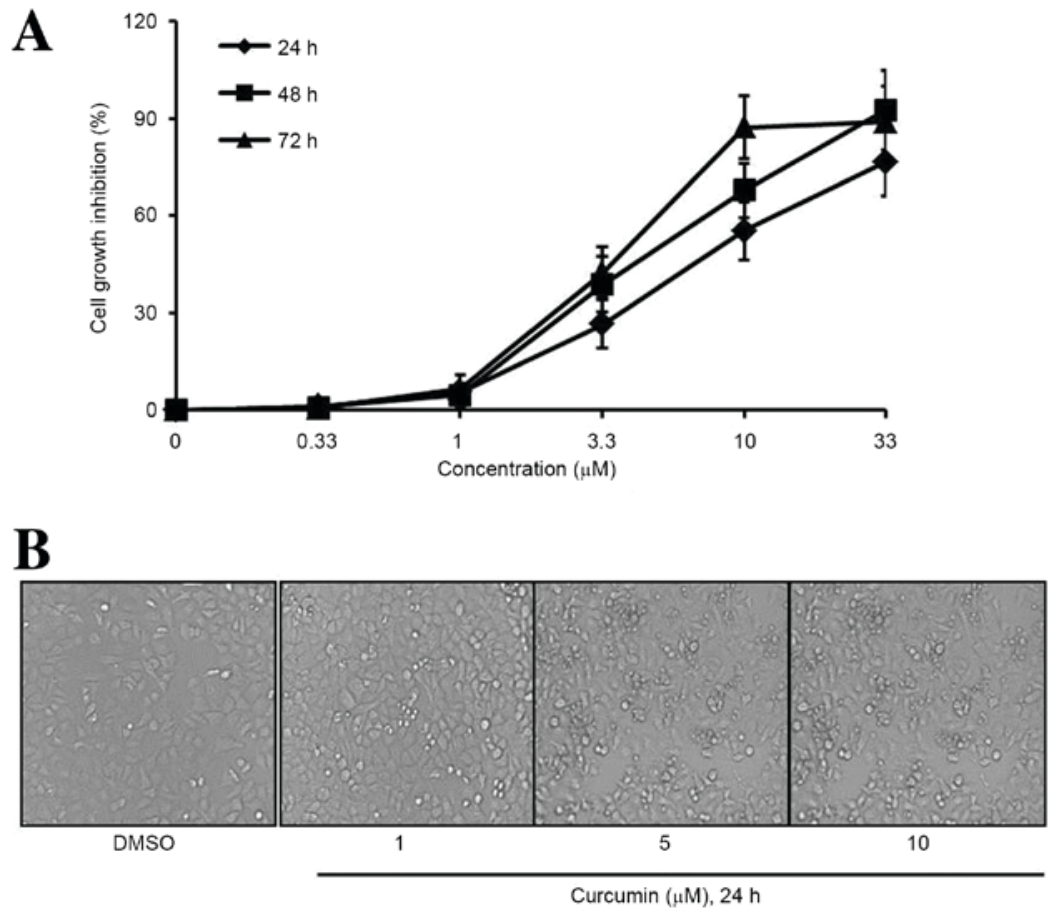

Figure 2. Curcumin decreases the viability of osteosarcoma cells. (A) Following treatment with $0,0.33,1,3.3,10$ or $33 \mu \mathrm{M}$ curcumin for 24,48 or $72 \mathrm{~h}$, cell viability was determined by a cell counting kit- 8 assay. Curcumin decreased cell viability in a time- and concentration-dependent manner. (B) Following treatment with DMSO, 1,5 or $10 \mu \mathrm{M}$ curcumin for $24 \mathrm{~h}$, cells were observed by a phase contrast microscope. Data are represented as the mean \pm standard deviation, $\mathrm{n}=3$.

A

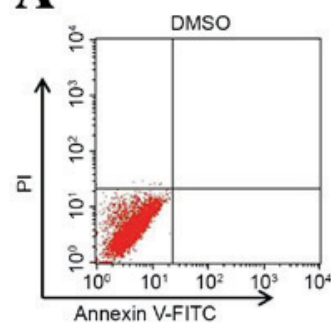

B

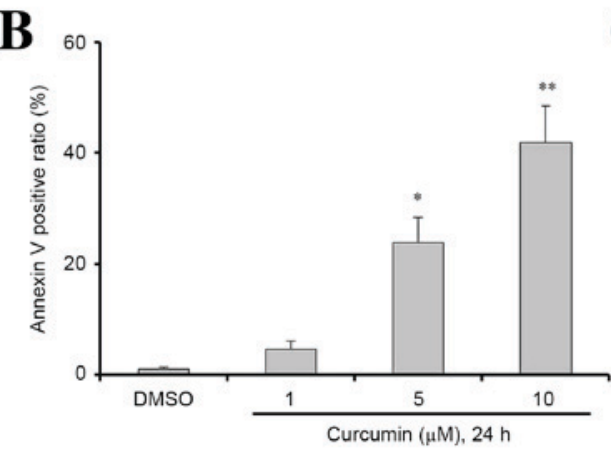

Curcumin $(\mu \mathrm{M}), 24 \mathrm{~h}$

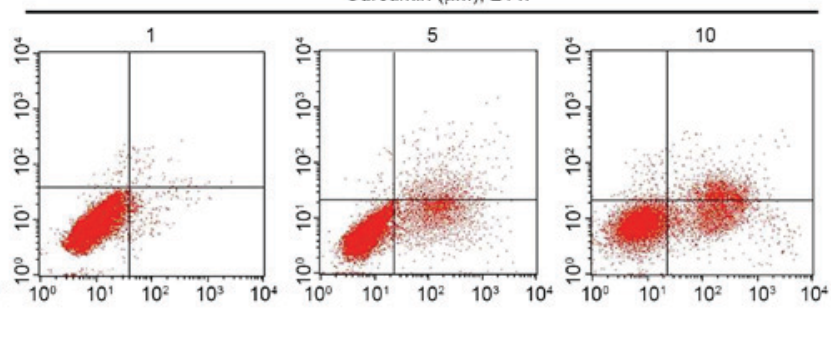

C

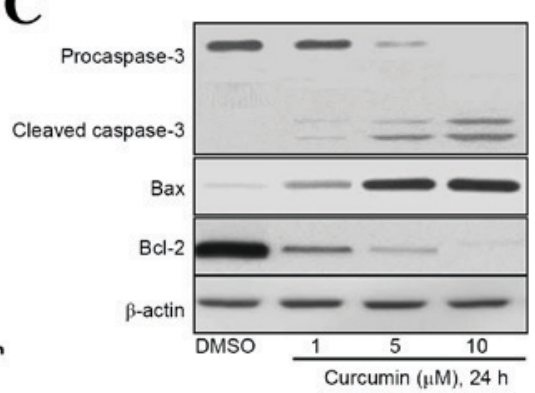

Figure 3. Curcumin induces osteosarcoma cells apoptosis. (A and B) MG63 cells were seeded at a density of $0.5 \times 10^{5}$ cells/dish in 60 -mm cell culture dishes and incubated for $24 \mathrm{~h}$. Cells were then treated with DMSO, 1, 5 or $10 \mu \mathrm{M}$ curcumin for $24 \mathrm{~h}$. Apoptosis was determined by a FITC-labeled Annexin V/PI apoptosis detection kit and flow cytometry. Representative results from flow cytometry are shown. (C) Western blot analysis was used to determine the expression levels of apoptosis-associated proteins in cells treated with DMSO or curcumin at different concentrations as indicated for $24 \mathrm{~h}$. Cell lysates were extracted for western blot analysis to determine the expression of procaspase-3, cleaved-caspase-3, Bcl-2, Bax, and $\beta$-actin (loading control). Data are presented as the mean \pm standard deviation, $\mathrm{n}=3$. ${ }^{*} \mathrm{P}<0.05$ and ${ }^{* * *} \mathrm{P}<0.01$ vs. the DMSO group. DMSO, dimethyl sulfide; Bcl-2, B-cell lymphoma 2; FITC, fluorescein isothiocyanate; PI, propidium iodide.

group (Fig. 3B; $\mathrm{P}<0.05$ and $\mathrm{P}<0.01$, respectively). To further investigate the potential mechanisms of curcumin-induced apoptosis in MG63 cells, the expression levels of apoptosis signaling proteins were detected by western blot analysis. The results indicated that the protein expression levels of cleaved caspase- 3 and Bax were increased, while the expression of 

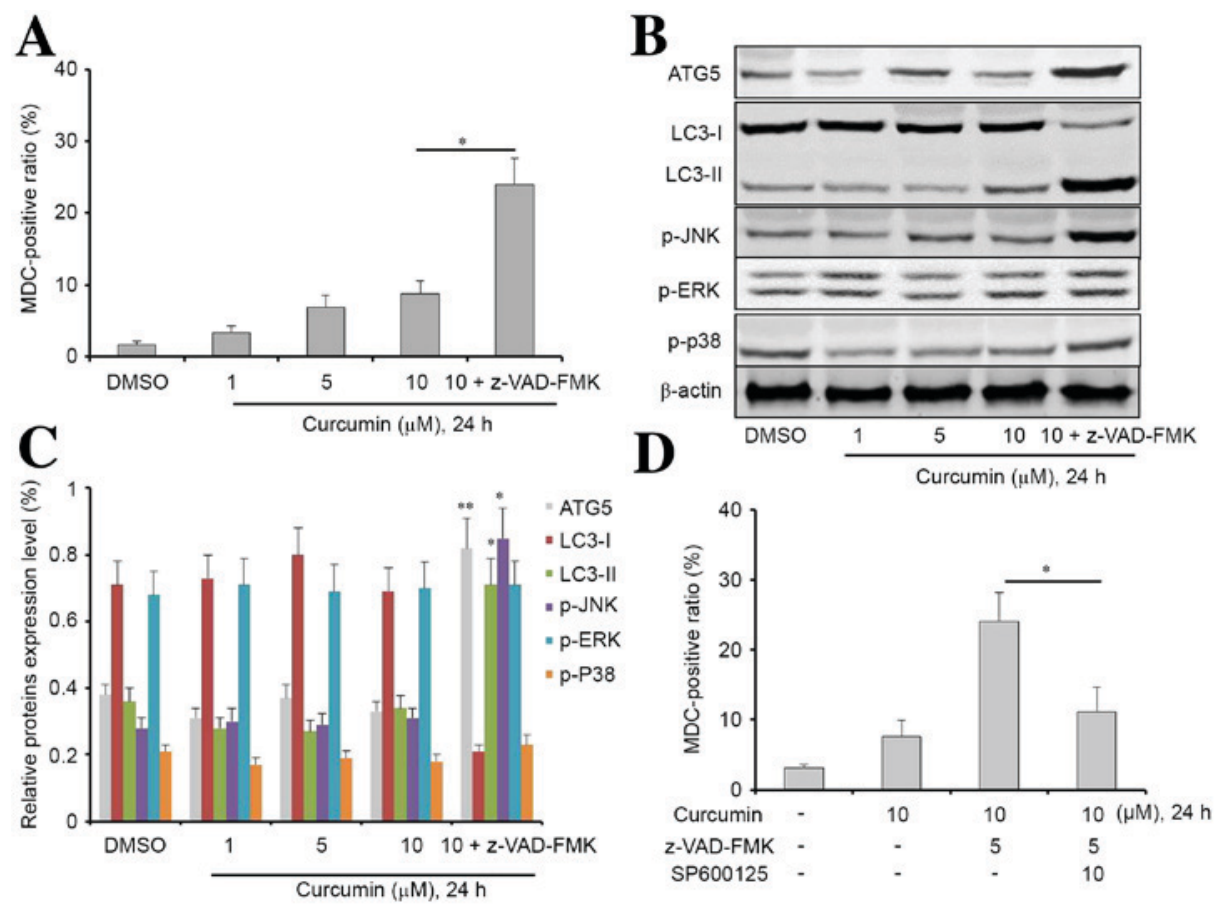

Figure 4. Z-VAD-FMK is beneficial to the induction of autophagy of curcumin-treated MG63 cells. (A) Following treatment with $1,5,10 \mu \mathrm{M}$ curcumin or $10 \mu \mathrm{M}$ curcumin combined with z-VAD-FMK for $24 \mathrm{~h}$, cell autophagy was determined by MDC assay. Curcumin increased cell autophagy in a concentration-dependent manner. In addition, z-VAD-FMK increased curcumin-induced autophagy in MG63 cells. (B and C) Western blot analysis was used to determine the expression levels of autophagy-associated proteins in cells treated with DMSO, curcumin at different concentrations or curcumin combined with z-VAD-FMK (as indicated) for $24 \mathrm{~h}$. Cell lysates were extracted for western blot analysis to assess the expression of ATG5, LC3-I, LC3-II, p-JNK, p-ERK, p-p38. $\beta$-actin was used as a loading control. ${ }^{*} \mathrm{P}<0.05$ and ${ }^{* *} \mathrm{P}<0.01$ vs. the DMSO group. (D) p-JNK inhibitor SP600125 turnover z-VAD-FMK increased curcumin-induced autophagy in MG63 cells. Data are presented as the mean \pm standard deviation, $\mathrm{n}=3$. ${ }^{*} \mathrm{P}<0.05$ and ${ }^{* *} \mathrm{P}<0.01$. MDC, monodansylcadaverine; ATG5, autophagy related 5; LC3-I, light chain 3-I; LC3-II, light chain 3-II; p-JNK, phosphorylated c-Jun N-terminal kinase 3; p-ERK, phosphorylated extracellular signal-regulated kinase.

anti-apoptosis proteins Bcl-2 and caspase-3 were reduced after treatment (Fig. 3C). These results indicate that the mechanism underlying curcumin-induced apoptosis may involve the activation of the caspase- 3 pathway in MG63 cells.

Inhibition of apoptosis enhances curcumin-induced autophagy through the JNK signaling pathway. The current study subsequently explored whether curcumin was able to alter the level of autophagy, which would consequently affect cell apoptosis. The auto-fluorescent substance MDC was used as a marker to detect the level of autophagy in MG63 cells. Following incubation with curcumin for $24 \mathrm{~h}$, the percentage of MDC-positive cells significantly increased in a dose-dependent manner, particularly in the curcumin combined with z-VAD-FMK group (Fig. 4A; $\mathrm{P}<0.05$ ). These results suggest that $\mathrm{z}$-VAD-FMK was able to promote curcumin-induced autophagy in MG63 cells.

Next, we extracted the total protein from MG63 cells incubated with different concentrations of curcumin or with the combination treatment for $24 \mathrm{~h}$. The expression levels of ATG5 LC3-I LC3-2, p-JNK, p-ERK, and p-P38 were analyzed by western blotting. As shown in Fig. 4B and C, compared with the other groups, there was a significant increase in the expression levels of ATG5 $(\mathrm{P}<0.01)$, LC3-II $(\mathrm{P}<0.05)$, and p-JNK $(\mathrm{P}<0.05)$ in the curcumin combined with $\mathrm{z}-\mathrm{VAD}-\mathrm{FMK}$ group. In addition, there was a significant reduction in the expression levels of LC3-I in the curcumin combined with z-VAD-FMK group compared with the other groups. Notably, JNK inhibitor
SP600125 effectively reversed combination treatment-induced autophagy in MG63 cells, suggesting that JNK pathway signaling may have an important role in curcumin-induced autophagy (Fig. 4D).

Inhibition of autophagy enhances curcumin-induced apoptosis in MG63 cells. Subsequently, in order to examine whether the inhibition of autophagy sensitizes MG63 cells to curcumin, cells were treated with curcumin, z-VAD-FMK or in combination with 3-MA, which is an autophagy inhibitor. Treatments with these drugs, either alone or in combination, significantly affected the apoptosis of MG63 cells $(\mathrm{P}<0.05)$. The results showed that 3-MA alone did not induce apoptosis or inhibit proliferation in MG63 cells (Fig. 5). However, an accumulation of apoptotic cells was observed upon treatment with curcumin, z-VAD-FMK and 3-MA, compared with treatment with curcumin and $\mathrm{z}-\mathrm{VAD}-\mathrm{FMK}$. This result indicated that curcumin-induced apoptosis was efficiently increased via inhibition of autophagy (Fig. 5A and B). Furthermore, 3-MA or SP600125 effectively reversed z-VAD-FMK-induced proliferation in curcumin-treated MG63 cells (Fig. 5C).

3-MA inhibits curcumin-induced hyperactive of p-JNK/autophagy pathway. To further explore the role of JNK in curcumin-induced autophagy, MG63 cells were used to investigate the effect of 3-MA on autophagic activity. As shown in Fig. 6, curcumin upregulated ATG5, p-JNK, and p53 expression levels in MG63 cells; however, z-VAD-FMK 

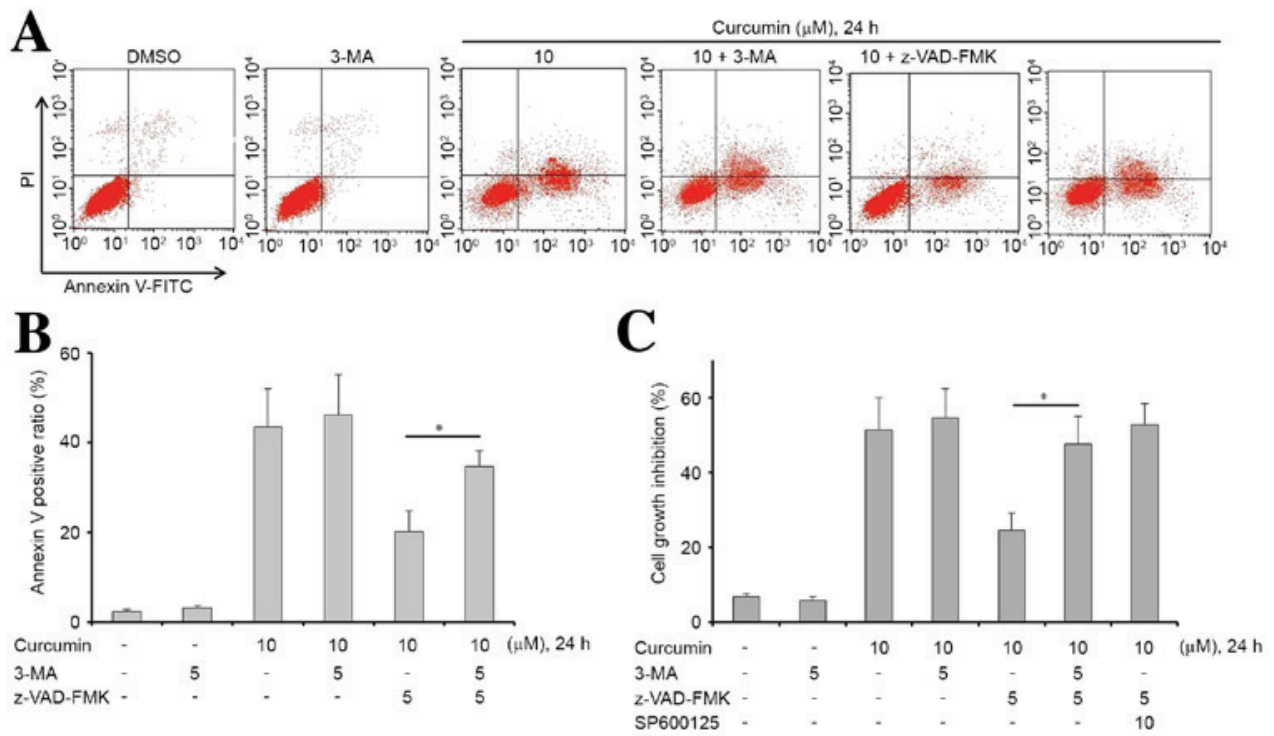

Figure 5.3-MA reversed z-VAD-FMK-induces apoptosis inhibition and proliferation in curcumin treated MG63 cells. (A and B) MG63 cells were treated with curcumin, z-VAD-FMK or in combination with 3-MA. Following treatment with these therapies for $24 \mathrm{~h}$, cell apoptosis was determined by flow cytometry. An accumulation of apoptotic cells was observed upon treatment with curcumin, z-VAD-FMK and 3-MA combined treatment compared with curcumin and z-VAD-FMK in MG63 cells. (C) Following treatment with these therapies for $24 \mathrm{~h}$, cell proliferation was determined by a cell counting kit-8 assay. 3-MA reversed z-VAD-FMK induced proliferation in curcumin treated MG63 cells. Data are presented as the mean \pm standard deviation, $\mathrm{n}=3$. ${ }^{*} \mathrm{P}<0.05$. FITC, fluorescein isothiocyanate; PI, propidium iodide; 3-MA, 3-methyladenine.
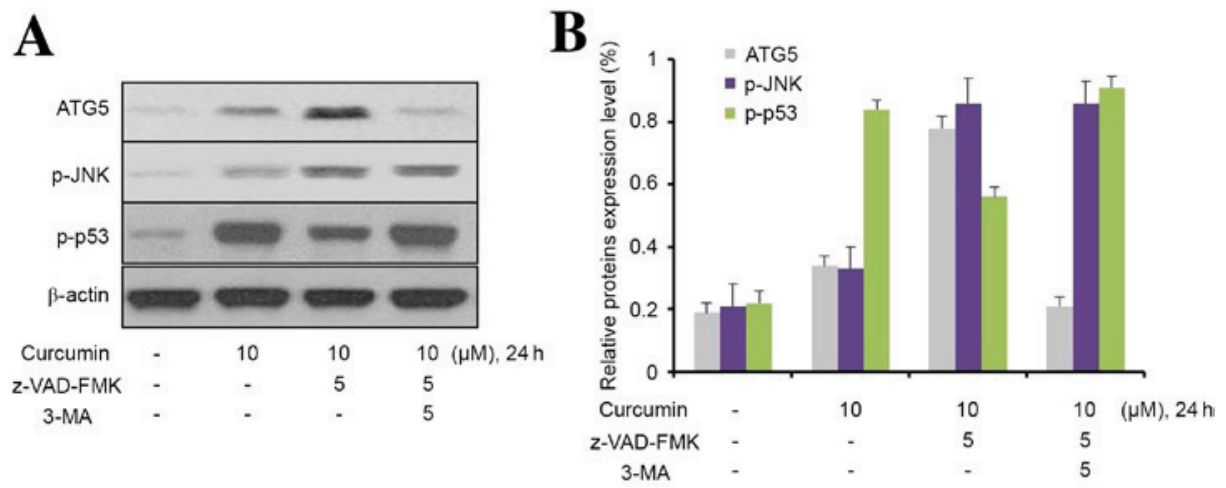

Figure 6. 3-MA inhibits curcumin-induced hyperactivity of the p-JNK/autophagy pathway. (A) Western blot analysis was used to determine the expression levels of autophagy-associated proteins in cells treated with DMSO, curcumin, Z-VAD-FMK and 3-MA, as indicated, for $24 \mathrm{~h}$. Cell lysates were extracted for western blot analysis to assess the expression levels of ATG5, p-JNK and p53. $\beta$-actin was used as a loading control. (B) Quantification of protein bands was performed using ImageJ software. Data are represented as the mean \pm standard deviation, $\mathrm{n}=3$. ATG5, autophagy related 5; p-JNK, phosphorylated c-Jun $\mathrm{N}$-terminal kinase; 3-MA, 3-methyladenine.

only upregulated the expression of ATG5 and p-JNK in 3-MA-treated cells. Furthermore, 3-MA effectively prevented the upregulation of ATG5 and p-JNK in MG63 cells induced by the combination of curcumin and z-VAD-FMK. Notably, z-VAD-FMK markedly downregulated p-P53 expression in curcumin-treated MG63 cells, and this phenomenon was inhibited by 3-MA.

\section{Discussion}

Given that osteosarcoma is characterized by adjuvant chemotherapy resistance and high rates of recurrence after curative resection (18), it is necessary to develop novel therapeutic agents to achieve improved patient prognoses. Curcumin is a natural compound derived from turmeric (Curcuma longa) and exhibits an effective antitumor effect on various cancers, including osteosarcoma (19). In addition, curcumin can reverse chemotherapy resistance in various types of human cancer $(7,20,21)$. Therefore, curcumin may be a promising agent for the treatment of osteosarcoma.

Several studies have confirmed that chemotherapy agents, including tamoxifen, 5-fluorouracil, and rapamycin, are able to induce autophagy (22-24). However, the mechanisms underlying autophagy in cancer therapy are complex and remain controversial. It has been reported that forced autophagy may be an apoptotic enhancer or a survival enhancer, depending on the experimental conditions $(22,25)$. Attempts have been made to elucidate the potential mechanisms involved in autophagy, so that it may be exploited as a target for cancer therapy. The current study showed that the expression levels of the anti-apoptotic protein Bcl-2 were significantly decreased in MG63 cells incubated with curcumin. The results of the present study suggest 
that inhibition of the Bcl-2-mediated anti-apoptotic pathway may contribute to curcumin-induced apoptosis in MG63 cells.

Autophagy has been confirmed to have two contrasting roles in cancer, in that it is both a tumor suppressor and a tumor protector (26). Recently, autophagy has been reported as a mechanism by which osteosarcoma cells develop resistance to anti-tumor agents, including cisplatin and doxorubicin $(27,28)$. Dysregulation of the p38 MAPK signaling pathway has an important role in tumor development and progression (29). In addition, p38 MAPK also mediates autophagy in response to anti-cancer agents in the treatment of cancer. Furthermore, p38 MAPK acts both as a positive and negative regulator in the autophagy process (29). JNK is initially activated in response to various stress signals and has been observed to participate in numerous cellular events including autophagy (30). The current study demonstrated that treatment with curcumin is correlated with increased phosphorylation of JNK in MG63 cells. JNK displays both tumor-promoting and tumor-suppressive functions depending on the genetic context of the tumor cells (31). The JNK pathway has been shown to be important in enforcing autophagy and apoptosis (32). Previous studies have further revealed that elevated JNK signaling is also associated with autophagy induction $(32,33)$. The present study also found that JNK inhibitor SP600125 was able to reverse curcumin-induced autophagy. These results suggest that the JNK pathway has a critical role in curcumin-induced autophagy.

The ERK signaling pathway has an important role in cancer development and progression. In addition, ERK activity has been confirmed to participate in autophagy and autophagic cell death (34). Notably, in human ovarian cancer cells, cytoplasmic sequestration of ERK by PEA-15 has been confirmed to promote autophagy (34). Furthermore, forced ERK activation by overexpression of activated MEK can promote autophagy without any other stimuli (35). However, there was no apparent change in p-ERK expression in osteosarcoma MG63 cells treated with curcumin, suggesting curcumin does not affect the ERK signaling pathway.

Autophagy and apoptosis are well-regulated biological processes that have important roles in tumor development and progression. The role of the anti-apoptotic protein $\mathrm{Bcl}-2$ in autophagy has been explored, and it has been proposed to be a major binding partner of Beclin-1, or to directly inhibit components of the autophagic pathway proteins, Bax and Bak (36,37). As previously demonstrated, p53 also participates in the regulation of autophagy. Nuclear p53 can induce autophagy through transcriptional effects, while cytoplasmic p53 may act as a master repressor of autophagy (38). It has been confirmed that p53 stimulates autophagy by transactivation of damage-regulated autophagy modulator or through the inhibition of the mTOR signaling pathway via activation of the AMP kinase $(39,40)$. Furthermore, the present results revealed that the autophagy inhibitor 3-MA was able to increase curcumin-induced apoptosis in MG63 cells. The present study also confirmed that 3-MA was able to reverse curcumin-induced upregulation of p-JNK and ATG5, a gene that is essential to the process of autophagy. In addition, 3-MA also upregulated curcumin-induced p53 expression. These results also ascertained that the JNK pathway has a critical role in curcumin-induced autophagy.
In conclusion, the present study demonstrated that curcumin inhibited the growth and induced the apoptosis of human osteosarcoma cells. The effects of curcumin-induced apoptosis in osteosarcoma cells were associated with caspase-3 activation and reduced the levels of $\mathrm{Bcl}-2$ expression. In addition, curcumin induces both apoptosis and autophagy in osteosarcoma cells. Furthermore, curcumin-induced autophagy has an anti-apoptotic role in osteosarcoma cells. These results provide important insight into the interaction between apoptosis and autophagy in osteosarcoma cells, and provides further information regarding the clinical treatment strategies using curcumin.

\section{References}

1. Anderson P and Salazar-Abshire M: Improving outcomes in difficult bone cancers using multimodality therapy, including radiation: Physician and nursing perspectives. Curr Oncol Rep 8: 415-422, 2006.

2. Isakoff MS, Bielack SS, Meltzer P and Gorlick R: Osteosarcoma: Current treatment and a collaborative pathway to success. J Clin Oncol 33: 3029-3035, 2015

3. Dieudonne FX, Marion A, Hay E, Marie PJ and Modrowski D: High Wnt signaling represses the proapoptotic proteoglycan syndecan-2 in osteosarcoma cells. Cancer Res 70: 5399-5408, 2010.

4. Yang J and Zhang W: New molecular insights into osteosarcoma targeted therapy. Curr Opin Oncol 25: 398-406, 2013.

5. Shakibaei M, Kraehe P, Popper B, Shayan P, Goel A and Buhrmann C: Curcumin potentiates antitumor activity of 5-fluorouracil in a $3 \mathrm{D}$ alginate tumor microenvironment of colorectal cancer. BMC Cancer 15: 250, 2015.

6. Hao F, Kang J, Cao Y, Fan S, Yang H, An Y, Pan Y, Tie L and Li X: Curcumin attenuates palmitate-induced apoptosis in MIN6 pancreatic $\beta$-cells through PI3K/Akt/FoxO1 and mitochondrial survival pathways. Apoptosis 20: 1420-1432, 2015.

7. Zhou Q, Ye M, Lu Y, Zhang H, Chen Q, Huang S and Su S: Curcumin improves the tumoricidal effect of mitomycin $\mathrm{C}$ by suppressing ABCG2 expression in stem cell-like breast cancer cells. PLoS One 10: e0136694, 2015.

8. van't Land B, Blijlevens NM, Marteijn J, Timal S, Donnelly JP de Witte TJ and M'Rabet L: Role of curcumin and the inhibition of NF-kappaB in the onset of chemotherapy-induced mucosal barrier injury. Leukemia 18: 276-284, 2004.

9. Yosifov DY, Kaloyanov KA, Guenova ML, Prisadashka K, Balabanova MB, Berger MR and Konstantinov SM: Alkylphosphocholines and curcumin induce programmed cell death in cutaneous T-cell lymphoma cell lines. Leuk Res 38: 49-56, 2014.

10. Zhou QM, Wang XF, Liu XJ, Zhang H, Lu YY, Huang S and Su SB: Curcumin improves MMC-based chemotherapy by simultaneously sensitising cancer cells to MMC and reducing MMC-associated side-effects. Eur J Cancer 47: 2240-2247, 2011.

11. Shanmugam MK, Rane G, Kanchi MM, Arfuso F, Chinnathambi A, Zayed ME, Alharbi SA, Tan BK, Kumar AP and Sethi G: The multifaceted role of curcumin in cancer prevention and treatment. Molecules 20: 2728-2769, 2015.

12. Gupta SC, Patchva S and Aggarwal BB: Therapeutic roles of curcumin: Lessons learned from clinical trials. AAPS J 15: 195-218, 2013.

13. Schmukler E, Kloog Y and Pinkas-Kramarski R: Ras and autophagy in cancer development and therapy. Oncotarget 5: 577-586, 2014.

14. Zhou YY, Li Y, Jiang WQ and Zhou LF: MAPK/JNK signalling: A potential autophagy regulation pathway. Biosci Rep 35: pii: e00199, 2015.

15. Zhang P, Zhang P, Zhou M, Jiang H, Zhang H, Shi B, Pan X, Gao H, Sun H and Li Z: Exon 4 deletion variant of epidermal growth factor receptor enhances invasiveness and cisplatin resistance in epithelial ovarian cancer. Carcinogenesis 34: 2639-2646, 2013.

16. Ma H, Chen H, Guo X, Wang Z, Sowa ME, Zheng L, Hu S, Zeng P, Guo R, Diao J, et al: M phase phosphorylation of the epigenetic regulator UHRF1 regulates its physical association with the deubiquitylase USP7 and stability. Proc Natl Acad Sci USA 109: 4828-4833, 2012. 
17. Hui B, Shi YH, Ding ZB, Zhou J, Gu CY, Peng YF, Yang H, Liu WR, Shi GM and Fan J: Proteasome inhibitor interacts synergistically with autophagy inhibitor to suppress proliferation and induce apoptosis in hepatocellular carcinoma. Cancer 118: 5560-5571, 2012

18. Wunder JS, Gokgoz N, Parkes R, Bull SB, Eskandarian S, Davis AM, Beauchamp CP, Conrad EU, Grimer RJ, Healey JH, et al: TP53 mutations and outcome in osteosarcoma: A prospective, multicenter study. J Clin Oncol 23: 1483-1490, 2005.

19. Chang Z, Xing J and $\mathrm{Yu} \mathrm{X}$ : Curcumin induces osteosarcoma MG63 cells apoptosis via ROS/Cyto-C/Caspase-3 pathway. Tumour Biol 35: 753-758, 2014.

20. Lu WD, Qin Y, Yang C, Li L and Fu ZX: Effect of curcumin on human colon cancer multidrug resistance in vitro and in vivo. Clinics (Sao Paulo) 68: 694-701, 2013.

21. Ye MX, Zhao YL, Li Y, Miao Q, Li ZK, Ren XL, Song LQ Yin $\mathrm{H}$ and Zhang J: Curcumin reverses cis-platin resistance and promotes human lung adenocarcinoma A549/DDP cell apoptosis through HIF-1 $\alpha$ and caspase-3 mechanisms. Phytomedicine 19: 779-787, 2012.

22. Pan X, Zhang X, Sun H, Zhang J, Yan M and Zhang H: Autophagy inhibition promotes 5 -fluorouraci-induced apoptosis by stimulating ROS formation in human non-small cell lung cancer A549 cells. PLoS One 8: e56679, 2013.

23. Li X, Wu D, Shen J, Zhou M and Lu Y: Rapamycin induces autophagy in the melanoma cell line M14 via regulation of the expression levels of Bcl-2 and Bax. Oncol Lett 5: 167-172, 2013.

24. Amaravadi RK, Yu D, Lum JJ, Bui T, Christophorou MA, Evan GI, Thomas-Tikhonenko A and Thompson CB: Autophagy inhibition enhances therapy-induced apoptosis in a Myc-induced model of lymphoma. J Clin Invest 117: 326-336, 2007.

25. Salazar M, Carracedo A, Salanueva IJ, Hernández-Tiedra S, Lorente $\mathrm{M}$, Egia $\mathrm{A}$, Vázquez $\mathrm{P}$, Blázquez $\mathrm{C}$, Torres $\mathrm{S}$, García S, et al: Cannabinoid action induces autophagy-mediated cell death through stimulation of ER stress in human glioma cells. J Clin Invest 119: 1359-1372, 2009.

26. Pandolfi PP: Breast cancer-loss of PTEN predicts resistance to treatment. N Engl J Med 351: 2337-2338, 2004

27. Miao XD, Cao L, Zhang Q, Hu XY and Zhang Y: Effect of PI3K-mediated autophagy in human osteosarcoma MG63 cells on sensitivity to chemotherapy with cisplatin. Asian Pac J Trop Med 8: 731-738, 2015

28. Chang Z, Huo L, Li K, Wu Y and Hu Z: Blocked autophagy by miR-101 enhances osteosarcoma cell chemosensitivity in vitro. ScientificWorldJournal 2014: 794756, 2014

29. Cuadrado A and Nebreda AR: Mechanisms and functions of p38 MAPK signalling. Biochem J 429: 403-417, 2010.
30. Kyriakis JM, Banerjee P, Nikolakaki E, Dai T, Rubie EA, Ahmad MF, Avruch J and Woodgett JR: The stress-activated protein kinase subfamily of c-Jun kinases. Nature 369: 156-160, 1994.

31. Wagner EF and Nebreda AR: Signal integration by JNK and p38 MAPK pathways in cancer development. Nat Rev Cancer 9: 537-549, 2009.

32. Wang C, Chen K, Xia Y, Dai W, Wang F, Shen M, Cheng P, Wang J, Lu J, Zhang Y, et al: N-acetylcysteine attenuates ischemia-reperfusion-induced apoptosis and autophagy in mouse liver via regulation of the $\mathrm{ROS} / \mathrm{JNK} / \mathrm{Bcl}-2$ pathway. PLoS One 9: e108855, 2014.

33. Davis RJ: Signal transduction by the JNK group of MAP kinases. Cell 03: 239-252, 2000

34. Cagnol S and Chambard JC: ERK and cell death: Mechanisms of ERK-induced cell death-apoptosis, autophagy and senescence. FEBS J 277: 2-21, 2010.

35. Corcelle E, Nebout M, Bekri S, Gauthier N, Hofman P, Poujeol P, Fénichel P and Mograbi B: Disruption of autophagy at the maturation step by the carcinogen lindane is associated with the sustained mitogen-activated protein kinase/extracellular signal-regulated kinase activity. Cancer Res 66: 6861-6870, 2006.

36. Pattingre S, Tassa A, Qu X, Garuti R, Liang XH, Mizushima N, Packer M, Schneider MD and Levine B: Bcl-2 antiapoptotic proteins inhibit Beclin 1-dependent autophagy. Cell 122: 927-939, 2005.

37. Lindqvist LM, Heinlein M, Huang DC and Vaux DL: Prosurvival Bcl-2 family members affect autophagy only indirectly, by inhibiting Bax and Bak. Proc Natl Acad Sci USA 111: 8512-8517, 2014.

38. Tasdemir E, Chiara Maiuri M, Morselli E, Criollo A, D'Amelio M, Djavaheri-Mergny M, Cecconi F, Tavernarakis N and Kroemer G: A dual role of p53 in the control of autophagy. Autophagy 4: 810-814, 2008.

39. Feng Z, Zhang H, Levine AJ and Jin S: The coordinate regulation of the p53 and mTOR pathways in cells. Proc Natl Acad Sci USA 102: 8204-8209, 2005.

40. Crighton D, Wilkinson S, O'Prey J, Syed N, Smith P, Harrison PR, Gasco M, Garrone O, Crook T and Ryan KM: DRAM, a p53-induced modulator of autophagy, is critical for apoptosis. Cell 126: 121-134, 2006 